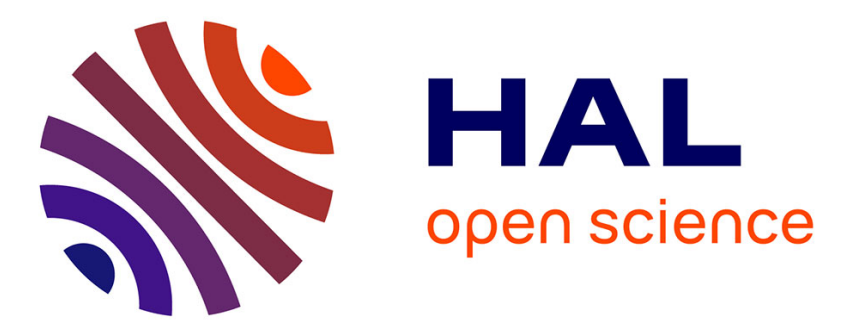

\title{
Non-destructive diagnosis of the integrity of green wood using ultrasonic computed tomography
}

Philippe Lasaygues, Emilie Franceschini, Eric Debieu, Loïc Brancheriau

\section{To cite this version:}

Philippe Lasaygues, Emilie Franceschini, Eric Debieu, Loïc Brancheriau. Non-destructive diagnosis of the integrity of green wood using ultrasonic computed tomography. International Congress on Ultrasonics, 2007, Vienne, Austria. CD-ROM(4 p.). hal-00440748

\section{HAL Id: hal-00440748 \\ https://hal.science/hal-00440748}

Submitted on 11 Dec 2009

HAL is a multi-disciplinary open access archive for the deposit and dissemination of scientific research documents, whether they are published or not. The documents may come from teaching and research institutions in France or abroad, or from public or private research centers.
L'archive ouverte pluridisciplinaire HAL, est destinée au dépôt et à la diffusion de documents scientifiques de niveau recherche, publiés ou non, émanant des établissements d'enseignement et de recherche français ou étrangers, des laboratoires publics ou privés. 


\title{
Non-destructive diagnosis of the integrity of green wood using ultrasonic computed tomography
}

\author{
Philippe Lasaygues ${ }^{1}$, Emilie Franceschini ${ }^{1}$, Eric Debieu ${ }^{1}$, Loïc Brancheriau ${ }^{2}$ \\ ${ }^{1}$ Laboratory of Mechanics and Acoustics, UPR CNRS 7051, 13402 Marseille cedex 20, France, lasaygues@1ma.cnrs-mrs.fr \\ ${ }^{2}$ Laboratory Production and Processing of Tropical Woods, UR CIRAD 40, 34398 Montpellier cedex 5, brancheriau@ cirad.fr
}

\begin{abstract}
Ultrasonic computed tomography (UCT) was used to assess the integrity of green wood. Woods are heterogeneous air-coupled, orthorhombic materials. Because of the difference in acoustic impedance between the material and the surrounding medium (water or coupling gel), the ultrasonic wave propagation is greatly perturbed by physical processes such as the refraction, attenuation and scattering of the waves. UCT belongs to the inverse scattering class of problems and the aim of this study was therefore to present a strategy for simply, efficiently and accurately developing a reconstruction algorithm. UCT is based on several assumptions, such as the presence of a low contrast medium (the biological medium), a large frequency range (a broadband pulse), and dense and complete sets of projections. If these conditions are ideally fulfilled, we can reconstruct images of the impedance of the medium. This technique involves an algorithm based on first-order Born approximation methods. To date we have tested the qualitative aspects of this imaging technique and part of the quantitative aspects by performing numerical simulations and on our tomographic testing ground. The latter is composed of a base supporting a rotating and translating mechanical structure that can hold several transducers, and a numerical system and computing and recording the projections. When performing non-destructive assessments on wood, we would have liked to obtain quantitative images related to acoustic parameter of wood (impedance, speed of sound, attenuation). However, since the problem is non-linear, a low-frequency method involving signal and image processing was used.
\end{abstract}

Key words: Ultrasonic Computed Tomography, Wood Imaging, simulation and experiments

\section{A. Introduction}

Acoustical properties such as the ultrasonic attenuation or velocity have been reported to reflect the strength of wood [1] and to predict in vivo decay processes [2]. However, the ultrasound methods currently available for assessing green wood status are limited to performing measurements in the transmission mode through a section. These methods therefore give average values recorded along the ultrasound pathway through the section. They are not suitable, however, for assessing the spatially heterogeneous acoustical properties of wood. The use of Ultrasonic Computed Tomography (UCT) in this context would constitute a significant step toward more accurate wood quality assessments. Although UCT imaging has been found to provide a valuable tool for imaging soft biological materials [3], little attention has been paid so far to the use of this method for imaging harder media such as green wood. L. Brancheriau et al. have published the preliminary results of an acoustic wave propagation study on green wood [4]. The interactions occurring between ultrasound and wood, especially in the case of green wood, make the imaging process particularly intricate. For instance, classical reconstruction algorithms are based on the assumption that straight ray propagation is involved, whereas the incident beam is liable to be refracted at the interface between the soft background (coupling gel, water) and the wood, depending on the incident angle, due to the great difference between the acoustic properties of the two media. Diffraction, which is closely related to refraction, can also result from the irregular shape of the samples. The anisotropy of the ultrasound propagation processes occurring through green wood can also sometimes require the use of sophisticated reconstruction tools.

Despite the many difficulties encountered when attempting to perform quantitative ultrasonic tomography on wood, the UCT imaging of wood provides an attractive non-invasive tool with which it is possible to perform quantitative 3-D reconstruction of the geometry of trees and determine acoustical properties such as the ultrasound velocity and attenuation, depending on the type of measurements (the time of flight or amplitude) performed. In previous studies by G. Nicolotti [5] and V. Bucur [6], projection data were obtained in the transmission mode through a wood specimen, which could be inversed using classical tomographic reconstruction algorithms [7]. Previously published preliminary results showed that the tomographic reconstruction of an academic test object and green wood was feasible, using ultrasonic transmission tomography techniques. The authors therefore hypothesized that the reconstruction of the outer boundary of the samples and the inner boundaries of the various regions of decay would provide information about the strength of the wood.

However, these studies were seriously limited by several factors. First, the boundaries of the object can't be easily reconstructed from the data set acquired in the transmission mode, but from data set acquired in the reflection mode, which gives an improved tomography of the shape of the scatterer. Furthermore, the mesh in transmission-UCT is very often too large, and the cavities of diameter lower than $30 \mathrm{~mm}$ are generally imperfectly 
identified. Secondly, inversing the data set acquired in the transmission mode to recover the sound velocity image of a cross-section requires the signals to be unambiguously identified, and the time-of-flight to be measured accurately from a signal with a low signal-to-noise ratio. The complex interactions between the incident ultrasonic wave field and wood give rise to a complex transmitted field with several output signals, which somehow makes it difficult to identify the signals.

The main objectives of our project were first to take full advantage of UCT in the diffraction and transmission modes by developing a technique for the cross-sectional imaging of wood, and secondly, to refine the signal processing technique by developing a suitable signal filtering process based on wavelet decomposition to improve the assessments based on time of flight measurements.

The latter point was addressed in a previous paper [8]. The present paper focuses on the qualitative (non parametric) image obtained using reflection-UCT to detect decay in a green wood. Reflection-UCT, which is a particular case of the general diffraction-UCT approach, consists in forming an image of the difference in contrast between the different layers of the wood (i.e. the shape imaging) from the projection data resulting from backscattering.

By performing a numerical simulation, we will study the effects of several patterns of decay and several configurations of "artificial" wood on the reflection-UCT data obtained. The experimental test performed on a green wood sample using a tomographic scanner showed the advantages and the limitations of the classical algorithms.

\section{B. Assumptions adopted in UCT}

Reflection-UCT can be used to detect (theoretically small) perturbations in the medium studied. In the case of soft, low- contrast media such as biological tissues, weak scattering approximations are generally used, such as the approximations of Born or Rytov [9].

Contrary to what occurs with soft media, the acoustical contrast between wood and the surrounding reference medium (coupling gel or water) is so great that the problem is intrinsically non-linear in terms of the unknown physical and geometrical parameters, and there generally exists no single solution.

One solution therefore consists in finding the homogenized or "equivalent" material parameters of a spatially bounded heterogeneous medium, and adapting the acquisition and reconstruction software initially developed for used with soft media.

Trees consist of long circular cylinders surrounded by an irregular thick bark. These cylinders are generally assimilated to orthotropic materials with a principal axis of symmetry oriented along the vertical axis [10]. In this study, the transversal cross-sections of the wood to be imaged were taken to be isotropic and were therefore assumed to mediate the propagation of more complex waves, similar to volume waves
Since the typical compression bulk wave velocity recorded in the transverse direction was $1400-1600 \mathrm{~m} / \mathrm{s}$, the wavelength at the frequency used here $(\sim 0.5 \mathrm{MHz})$ was of the order of 2.8 to 3.2 millimeters, which is much larger than the usual structural heterogeneities $(\sim 10$ micrometers). Wood can therefore be said to be a homogeneous medium on the scale of the wavelength and the Born approximation can be used.

The wavelength in water $(\sim 3 \mathrm{~mm})$ was much shorter than the diameter of the object (approximately $100-200$ $\mathrm{mm}$ ). Ray theory was applied and straight lines inside the object were taken to approximate the wave propagation paths. Since only signal contributions associated with compression waves were of interest here, a weakly inhomogeneous fluid can be used to model wood.

\section{Experimental set-up}

The experimental setup was designed to perform all UCT-measurements (in the reflection, diffraction and/or transmission modes). In included an acoustic device, which could be moved with various degrees of freedom and used to analyze the samples in all directions. The position of the target and the transducers could be adjusted. In particular, the operator could prescribe the exact positions of the transmitter and the receiver. During all the measurements, the wave velocity in the water tank was $1480 \mathrm{~m} / \mathrm{s}$.

Ultrasounds were generated using a Panametric ${ }^{\circledR}$ pulse/receiver and Imasonic ${ }^{\circledR}$ piezo-composite wide-band transducers with a nominal frequency of $0.5 \mathrm{MHz}$. The object under investigation was placed in the centre of the measuring system. The sector scanned was typically $72^{\circ} /$ $360^{\circ}$, (i.e. 72 view angles), and the angular increment used was $5^{\circ}$. The typical backscattered signals were composed of 4096 samples regularly spaced at intervals of $50 \mathrm{nsec}(20 \mathrm{MHz})$. In the reflection mode, the projections corresponded to the backscattering echoes and to the complete Radio-Frequency signal. The reconstruction algorithm was the algorithm of the UCT based on the summation and the spreading of the filtered projections. The size of the images was 300 x 300 pixels.

\section{Results}

Reflection-UCT was applied to a numerically simulated test-target and to a green wood sample.

The numerically simulated test-target was a homogeneous and isotropic circular wood cylinder with a diameter of $30 \mathrm{~mm}$ (see Fig.1-a). The mean velocity of the compressional wave was $1500 \mathrm{~m} / \mathrm{s}$ in water, and 1600 $\mathrm{m} / \mathrm{s}$ in the wood. The sample contained two inclusions corresponding to decay $\left(c_{1}=700 \mathrm{~m} / \mathrm{s}\right.$ and $\left.\mathrm{c}_{2}=1300 \mathrm{~m} / \mathrm{s}\right)$.

The spruce wood sample (picea abies) was green, fresh, streamlined, surrounded by bark, and measured 125 $\mathrm{mm}$ in diameter (see Fig.2-a). The mean velocity of 1400 $\mathrm{m} / \mathrm{s}$ measured at the center of a piece of wood with plane parallel faces from the same specimen, was used as the required a priori value. A mixture of glue and sawdust was placed in a $30 \mathrm{~mm}$-hole drilled in the center of the sample. 


\section{D.1. Simulation test}

Fig.1-b gives the ultrasonic sinogram and fig.1-c, the reflection-UCT of the numerically simulated test-target.

This image was geometrically accurate as far as the actual dimension of the object was concerned.

The result was acceptable, since the Born approximation was used and the background consisting of water with an a priori wave velocity equal to $1500 \mathrm{~m} / \mathrm{s}$. The perturbation in this case was introduced by the two holes simulating decay. The most highly contrasted area therefore naturally focused on the inner holes rather than on the external boundaries of the target. In fact, in this example, the bark was not simulated, and the difference in velocity between the water and the wood was very insignificant $(100 \mathrm{~m} / \mathrm{s})$. It was not in fact possible for the algorithm to discriminate between the two domains. The reference background used for the inversion procedure was finally the set composed of the water and the main wood. Since the difference in velocity between the wood and the holes increased, they could easily be identified in this example.

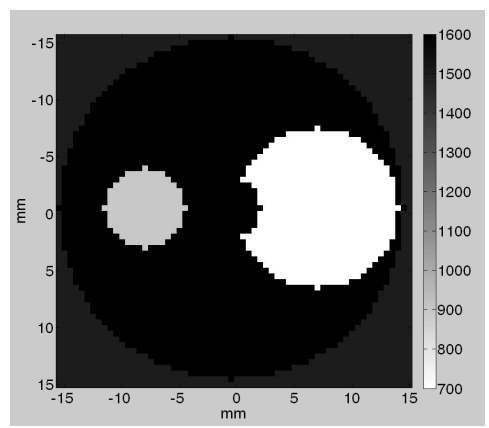

(a)

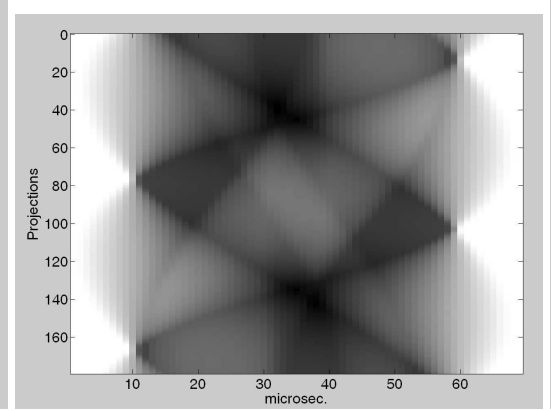

(b)

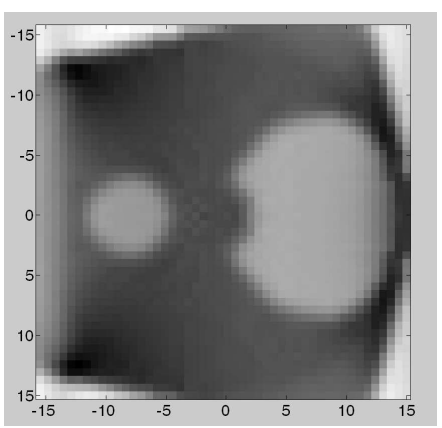

(c)

Fig.1. Numerically simulated test-target consisting of a homogeneous and isotropic circular wood cylinder 30 mm in diameter. (a) Sample; (b) ultrasonic reflection sinogram; (c) tomogram

\section{D.2. Wood specimen}

Fig. 2 shows the reconstructed reflection-UCT of a spruce wood sample (picea abies). The difficulties encountered were mainly due to the strong attenuation of the waves occurring during their propagation. Signals were therefore amplified but not saturated.

Besides the attenuation of the waves during the propagation, it is worth mentioning that the effects of the bark on the signals were of great importance. Large amounts of air are probably imprisoned between the healthy tree and the bark. The acoustic impedances are very different and this affects the wave propagation by brutal interrupting. Given the irregularity of the surface texture, it is likely that only a small part of the incident energy will penetrates into the wood, and a large proportion will be directly backscattered without penetrating into the healthy wood. This idea is in line with the strong echoes, which, if we assume that they are not attenuated between the healthy wood and the bark, can be taken to reflect the thickness of the bark.

The diameter of the wooden sample was correctly assessed $(125 \mathrm{~mm})$, and the cavity was positioned well in the center, but it was impossible to measure its diameter exactly. Here again, the problem is due to the low contrast in the sound speed between the region of interest and the rest of the sample. In addition, it was necessary to assume (as we did at the beginning) that we were dealing with isotropic conditions.

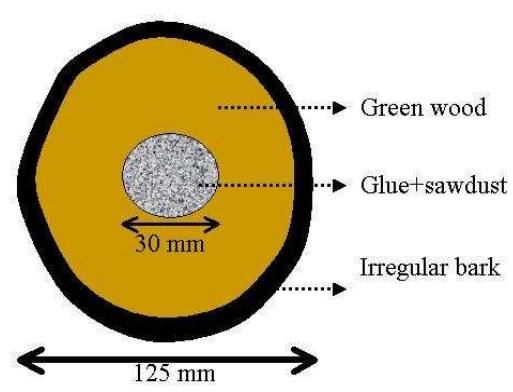

(a)

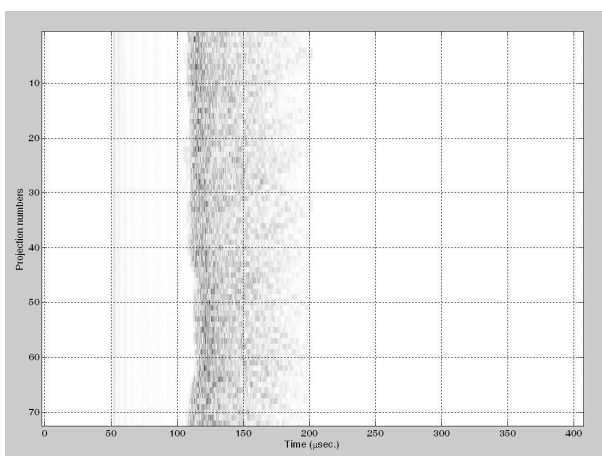

(b)

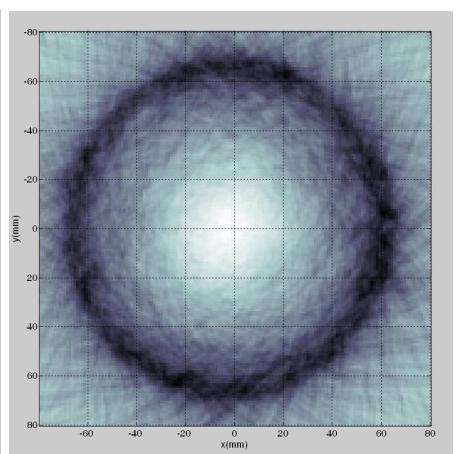

(c) 


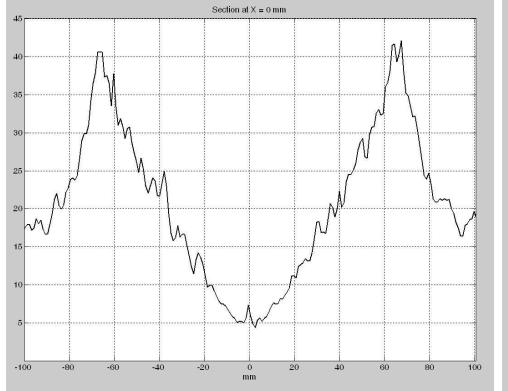

(d)

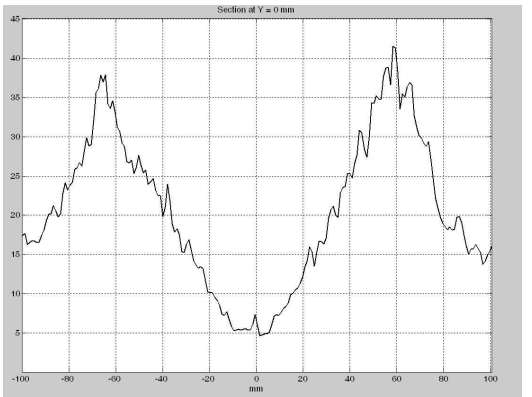

(e)

Fig.2. Reflection-UCT of spruce wood sample (picea abies) (a) geometry (b) ultrasonic reflection sinogram; (c) tomogram; (d-e) $\mathrm{x}$ and $\mathrm{y}$ pixel profiles drawn at $\mathrm{x}=\mathrm{y}=0 \mathrm{~mm}$

\section{E. Conclusion}

Here we have presented wood ultrasonic imaging method based on the use of Ultrasonic Computed Tomography. Due to the acoustical properties of wood (the attenuation and impedance contrast), working with wood is more complex than dealing with soft tissues, which made it necessary to adapt the linear inversion procedure usually applied to the data, using a linear approximation of the direct problem, the Born approximation. It was necessary to study the non-linear inverse problem. But in our case, wood was assumed to be equivalent to an internally weakly contrasted object (the local fluctuations in the acoustic characteristics of a cross-section are weak) immersed in a homogeneous reference medium (water). An ultrasonic tomography procedure was then applied. The results of the reconstructed geometry are similar to the real values.

This research could now be continued on two possible lines. The first approach is based on the Born iterative method, where the iterations are calculated numerically from experimental data [11]. The second possible approach is based on modeling the forward problem using the Intercepting Canonical Body Approximation method, which reduces the cost of the calculations. The latter method consists in taking the wood covered with bark and the decay it contains to be a non-circular multi-layer cylinder, in which every layer (bark, wood, decay) is homogeneous. Contrary to approaches based on straight ray theory, this method requires no limited approximations and no a priori information. The scattered field is completely integrated [12].

\section{F. Literature}

[1] V. Bucur, "Acoustic properties of wood in tridimensional representation of slowness surfaces," Ultrasonics, vol. 40 (1-8), pp. 537, 2002

[2] J. I. Dunlop, "Testing of poles by using acoustic pulse method," Wood Science and Technology, vol. 15 (4), pp. 301-310, 1981

[3] S. Mensah and J.P. Lefebvre, "Enhanced Diffraction Tomography," IEEE Trans. Ultrason. Ferroelec. Freq. Control, Vol 44, (6), pp 1245,1252, 1997

[4] A. Dikrallah, A. Hakam, B. Kabouchi, L. Brancheriau, H. Baillères, A. Famiri, M. Ziani, "Experimental analysis of acoustic anisotropy of green wood by using guided waves," ESWM-COST Action E35, 2004

[5] G. Nicolotti, L.V Socco, R. Martinis, A. Godio, and L. Sambuelli, "Application and comparison of three tomographic techniques for detection of decay in trees," Journal of Arboriculture, vol. 29 (2), pp. 66-77, 2003

[6] V. Bucur, Acoustics of wood, CRC Press Inc., 1995

[7] A.C. Kak and M. Slaney, "Principles of computerized tomographic imaging," IEEE press, 1999

[8] P. Lasaygues, "Wavelet based deconvolution method in ultrasonic tomography," Proceeding of the International Congress on Ultrasonics, be appear, 2007

[9] F. Greenleaf, "Introduction to Computer Ultrasound Tomography," Computed Aided Tomography and Ultrasonics in Medicine, North-Holland, pp. 125-136. 1970

[10] J.R. Goodman and J. Bodig, "Orthotropic Elastic Properties of Wood," Journal of the Structural Division, vol. 96 (11), pp. 2301-2319, 1970

[11] P. Lasaygues, R. Guillermin and J.-P. Lefebvre, "Distorted Born Diffraction Tomography: limits and applications to inverse the ultrasonic field scattered by an non-circular infinite elastic tube," Ultrasonic Imaging, vol. 28, pp. 211-229, 2006

[12] E. Franceschini, S. Mensah, L. Le Marrec and P. Lasaygues, "An optimization method for quantitative impedance tomography," IEEE Trans. Ultrason. Ferroelec. Freq. Control, - Special Issue on High Resolution Ultrasonic Imaging in Industrial, Material and Biomaterial Applications, in press., 2006 